\section{Bessere Lebensqualität und Kosteneffektivität nach Laserablation bei Varikose}

Brittenden J, Cooper D, Dimitrova $\mathrm{M}$ et al. FiveYear Outcomes of a Randomized Trial of Treatments for Varicose Veins. N Engl J Med 2019; 381: 912-922

Minimalinvasive Behandlungsoptionen wie die endovenöse Laserablation und die ultraschallgesteuerte Schaumsklerosierung sind mittlerweile empfohlene Alternativen zur Operation bei der Behandlung von Krampfadern. Mittelfristige Follow-up-Studien zeigen, dass die Laserablation, die Schaumsklerosierung und die Operation in äquivalenten Rezidivraten und Lebensqualität nach 2 und 3 Jahren resultieren. Jedoch bleibt die Langzeiteffektivität der Verfahren im Vergleich bislang unklar.

Eine Gruppe von Autoren um Brittenden aus Glasgow untersuchte in ihrer randomisierten kontrollierten Studie mit 798 Teilnehmern die Langzeitwirkung hinsichtlich Lebensqualität und Kosteneffektivität. Dazu schlossen sie Patienten aus 11 Zentren im Vereinigten Königreich ein. Die primären Endpunkte nach 5 Jahren waren die krankheitsspezifische und allgemeine Lebensqualität sowie die Kosteneffektivität auf Basis der erwarteten Kosten und der QALYs (quality-adjusted life-years), ermittelt durch Behandlungskosten und den Scores gemäß dem EuroQol-EQ-5D-Fragebogen.

\section{Ergebnisse}

Die Lebensqualitätsfragebögen wurden von 595 (75\%) der 798 Teilnehmer ausgefüllt. Nach Adjustierung für die Ausgangswerte und andere Variablen waren die Scores nach dem Aberdeen-Varicose-Vein-Questionnnaire geringer bei Patienten, die einer Laserablation oder chirurgischen Therapie unterzogen wurden, als unter denen, die die Schaumsklerotherapie erhalten hatten (Effektgröße Laserablation vs. Schaumsklerotherapie $-2,86$; $95 \%-K I$ : $-4,49$ bis $-1,22$; $\mathrm{p}<0,001$; Operation vs. Schaumsklerotherapie: $-2,6$; 0,95\%-KI: $-3,99$ bis $-1,22$; $\mathrm{p}<0,001)$. Die allgemeine Lebensqualität unterschied sich nicht in den Gruppen. Die Kosten-Effektivitäts-Analyse favorisierte die Laserablation. Im Vergleich zwischen Schaumsklerotherapie und Operation favorisierten 54,5\% der Modelliterationen die Operation.

\section{FAZIT}

In ihrem großen Multicenter-Trial konnten die Autoren zeigen, dass sich in allen 3 Gruppen die Lebensqualität nach 5 Jahren deutlich verbessert hatte. Es gab jedoch bedeutsame klinische Unterschiede in den Gruppen hinsichtlich der krankheitsspezifischen Lebensqualität, was die Laserablation und die Operation gegenüber der Schaumsklerotherapie favorisierte. Die Laserablation war der Operation in Hinblick auf die Lebensqualität gleichrangig und hatte unter allen 3 Behandlungsmethoden die höchste Chance, kosteneffektiv zu sein.

MOR Dr. med. Benedikt Lampl, Regensburg 